Rev. Int. Contam. Ambie. 37, 589-599, 2021

https://doi.org/10.20937/RICA.54008

\title{
EFECTO DEL TAMAÑO DE PARTÍCULA DE DIÓXIDO DE TITANIO INMOVILIZADO EN LA OXIDACIÓN FOTOCATALÍTICA DE PÚRPURA DE BROMOCRESOL
}

Effect of titanium's dioxide particle size immobilized on the photocatalytic oxidation of purple of bromocresol

\author{
Mario Alberto ALVARADO-DE LA VEGA ${ }^{1}$, Rocío MEZA-GORDILLO ${ }^{1}$, \\ Juan José VILLALOBOS-MALDONADO ${ }^{1}$, José Francisco POLA-ALBORES ${ }^{2}$ \\ y Samuel ENCISO-SÁENZ ${ }^{1 *}$
}

${ }^{1}$ Tecnológico Nacional de México/Instituto Tecnológico de Tuxtla Gutiérrez, Laboratorio 5, Polo Tecnológico, km 1080 Carretera Panamericana, 29050 Tuxtla Gutiérrez, Chiapas, México.

${ }^{2}$ Instituto de Investigación e Innovación en Energías Renovables, Universidad de Ciencias y Artes de Chiapas, Libramiento Norte Poniente 1150, Colonia Lajas Maciel, 29039 Tuxtla Gutiérrez, Chiapas, México.

*Autor para correspondencia: senciso@ittg.edu.mx; samenciso@hotmail.com

(Recibido: mayo 2020; aceptado: diciembre 2020)

Palabras clave: colorante, fotocatálisis, semiconductor, revestimiento por inmersión, catalizador

\section{RESUMEN}

La contaminación de agua residual con materia orgánica y compuestos aromáticos es un problema cada vez más complejo que demanda sistemas de tratamiento más eficientes. El objetivo de este trabajo es evaluar el efecto del tamaño de partícula de $\mathrm{TiO}_{2}$ en la oxidación fotocatalítica del púrpura de bromocresol usado como modelo de contaminante orgánico. $\mathrm{El} \mathrm{TiO}_{2}$ se inmovilizó sobre un sustrato de borosilicato mediante la técnica de revestimiento por inmersión y se caracterizó por microscopia de fuerza atómica (AFM, por sus siglas en inglés) para evaluar la morfología de la superficie obtenida. La estructura cristalina anatasa del $\mathrm{TiO}_{2}$ se confirmó mediante difracción de rayos X (DRX) y el tamaño de partícula se determinó mediante dispersión de luz dinámica (DSL). Se evaluaron los efectos de partículas de 25 y $300 \mathrm{~nm}$ de $\mathrm{TiO}_{2}$ sobre la degradación del púrpura de bromocresol en concentraciones de 5, 10, 20 y 30 ppm. La cinética de la reacción de degradación de púrpura de bromocresol (PBC) fue de pseudoprimer orden, congruente con el modelo de Langmuir para concentraciones bajas de este colorante. La fotodegradación en partículas de $25 \mathrm{~nm}$ de $\mathrm{TiO}_{2}$, resultó más eficiente que la de $300 \mathrm{~nm}$, debido al mayor número de granos formados en la superficie, el cual promovió un mayor número de sitios activos disponibles.

Key words: stain, photocatalysis, semiconductor, cladding by immersion, catalyst

\begin{abstract}
Pollution of sewage water with organic matter and aromatic compounds is a complex problem that is constantly in need of more efficient systems for its treatment. The objective of this works is to evaluate the effect of $\mathrm{TiO}_{2}$ particle size in the photocatalytic oxidation of bromocresol purple, used as a model of organic polluter. $\mathrm{TiO}_{2}$ was immobilized on a borosilicate substrate through the coating by immersion technic and was characterized by atomic force microscopy (AFM) to evaluate the morphology of
\end{abstract}


the obtained surface. The anatase crystalline structure of $\mathrm{TiO}_{2}$ was confirmed by $\mathrm{X}$ ray diffraction (DRX) and its particle size was evaluated by dynamic light scattering (DSL). The effects of 25- and $300-\mathrm{nm} \mathrm{TiO}_{2}$ particles on the degradation of bromocresol purple at 5, 10, 20 and $30 \mathrm{ppm}$ concentrations were evaluated. Kinetics of the bromocresol purple (PBC) degradation reaction was pseudo first order, consistent with the Langmuir model for low concentrations of this dye. The photodegradation in $25-\mathrm{nm} \mathrm{TiO} 2$ particles was more efficient than in $300 \mathrm{~nm}$, due to the greater number of grains formed on the surface, thus promoting a greater number of available active sites.

\section{INTRODUCCIÓN}

El agua residual generada en la industria causa graves problemas al medio ambiente, dado que con frecuencia contienen componentes orgánicos tóxicos difíciles de degradar biológicamente, los cuales representan un peligro para la vida acuática (Habibi y Mosavi 2017, Rizzi et al. 2017). Se han desarrollado varios tratamientos para la remediación de agua contaminada, como adsorción, floculación, precipitación, desinfección, separación con membranas y procesos de oxidación avanzada (Monga y Basu 2019, Yang et al. 2020). Los pigmentos que contaminan las aguas residuales evitan el paso de luz y afectan el oxígeno disuelto, los cuales son esenciales para la vida acuática, además de que grupos como el azoico, el trifenilmetano y la antraquinona, entre otros, poseen una compleja estructura molecular aromática, por lo que su degradación biológica es difícil (Bousnoubra et al. 2017, Momina et al. 2018, Deng et al. 2019, Talebi et al. 2019). Dentro de las opciones desarrolladas y utilizadas para el tratamiento de pigmentos se encuentran la floculación/coagulación, la ozonación, la electrocatálisis y la adsorción, siendo esta última la más utilizada, aunque con limitantes por costo y capacidad adsorbente del material usado (Zhang et al. 2020). De las $700000 \mathrm{t}$ de colorantes producidas al año a nivel mundial, del 2 al $50 \%$ son desechadas en los residuos como parte del proceso de manufactura, de las cuales $1000 \mathrm{t}$ provienen de la industria textil (Ali et al. 2019, González-Casamachin et al. 2019, Li et al. 2019).

En la actualidad se emplean varios métodos físicos, químicos y biológicos como coagulación, floculación, adsorción, electrólisis, y tratamientos aeróbicos y anaeróbicos, entre otros, para tratar el agua residual coloreada; sin embargo, los altos costos de operación demandan nuevos métodos de transformación (Das et al. 2017). Los procesos de oxidación avanzada, específicamente la oxidación fotocatalítica, parecen ser los más adecuados por su efectividad para destruir las estructuras cromofóricas de los colorantes y por su bajo consumo de energía (Gupta et al. 2012, Binaeian et al. 2016, Wang et al. 2020). Actualmente hay un interés creciente para el desarrollo de materiales que posean suficiente fotosensibilidad y puedan ser útiles para la resolución de problemas ambientales (Gora et al. 2018, Lu et al. 2018, Maghsoodi et al. 2019, Yao et al. 2020). $\mathrm{El} \mathrm{TiO}_{2}$ es un fotocatalizador ampliamente utilizado, ya que tiene ventajas como bajo costo, ausencia de toxicidad y estabilidad química; además, es adecuado para el tratamiento de contaminantes orgánicos en aire y agua como colorantes, fármacos, compuestos orgánicos clorados, compuestos fenólicos y herbicidas, entre otros (Du et al. 2015, Khatibnezhad y FaghihiSani 2015, Chen et al. 2016, Bagheri et al. 2017, Odling et al. 2018, Peleyeju y Arotiba 2018). $\mathrm{El} \mathrm{TiO}_{2}$ se activa mediante irradiación ultravioleta, generando el transporte de cargas del par electrón-hueco, las cuales son altamente oxidantes de contaminantes orgánicos. La formación de cargas promueve radicales $\mathrm{O}_{2}{ }^{-} \mathrm{y}$ ${ }^{\circ} \mathrm{OH}$, mineralizando los contaminantes orgánicos a $\mathrm{CO}_{2}$ y agua (Kment et al. 2015, Shinde et al. 2017).

Los revestimientos nanocristalinos de $\mathrm{TiO}_{2}$ son de especial interés científico y tecnológico por su aplicación potencial en dispositivos ópticos fotoelectrónicos, celdas solares, sensores de gas, tratamiento de agua, revestimientos de autolimpieza y fotocatálisis (Malnieks et al. 2016). Se han desarrollado varias técnicas para inmovilizar el catalizador sobre diferentes materiales de soportes tales como vidrio, zeolitas, metales y fotoelectrodos. Las más comunes son revestimiento por inmersión, descomposición hidrotérmica asistida por polímero, fotograbado, fundición con disolvente y pirolisis por aspersión (Srikanth et al. 2017). Para una adherencia suficientemente fuerte del revestimiento sobre el substrato, el método de preparación generalmente requiere de una etapa de alta temperatura después del revestimiento con la solución precursora (Vaez et al. 2012).

El tamaño de partícula es una de las características importantes de un catalizador, ya que define el área de superficie disponible para la adsorción y 
descomposición de contaminantes orgánicos. Es difícil evitar la agregación de partículas primarias para formar partículas secundarias que pueden afectar las propiedades de dispersión de luz del catalizador, así como el grado de penetración de fotones en éstas. Las propiedades de transporte de los reactantes y productos también pueden alterar la efectividad del catalizador (Henderson 2011, Pellegrino et al. 2017). En la investigación sobre la reducción fotocatalítica de $\mathrm{CO}_{2}$ (Koci et al. 2009), se determinó que el tamaño óptimo de la partícula depende de los efectos competitivos del área de superficie específica, la dinámica de flujo de cargas y la eficiencia de absorción de luz. Es importante comprender los factores que relacionan las propiedades con el tamaño de partículas de semiconductores, así como la inyección electrónica a éstos para entender el proceso de transferencia electrónica en fotocatálisis (Nam et al. 2018).

El tamaño de partícula y la actividad fotocatalítica son controladas también por otros parámetros como la concentración de grupos $\mathrm{OH}^{-}$inicialmente presentes en la superficie del catalizador y la disponibilidad de huecos fotogenerados. Estos parámetros tienen una relación explicada por la ecuación de la cinética, donde la constante de velocidad efectiva, $K_{\text {eff, es una }}$ función de la concentración de grupos hidroxilos, de huecos y de $k$, constante para las muestras a pesar de la diferencia en la calidad cristalina:

$$
K_{\text {eff }}=\mathrm{k}[\mathrm{OH}]\left[\mathrm{h}^{+}\right]
$$

donde $[\mathrm{OH}]$ se reduce como función del incremento del tamaño de la partícula, el cual es equilibrado por un incremento en $\left[\mathrm{h}^{+}\right]$en partículas mayores de 15 nm (Carneiro et al. 2010).

El objetivo de este trabajo se centra en demostrar el efecto del tamaño de partícula de $\mathrm{TiO}_{2}$, inmovilizado mediante el método de revestimiento por inmersión, sobre la oxidación fotocatalítica de contaminantes orgánicos con estructura molecular aromática, usando como modelo el púrpura de bromocresol.

\section{MATERIALES Y MÉTODOS}

\section{Impregnación de $\mathrm{TiO}_{2}$ en tubos de borosilicato}

Se emplearon tubos de vidrio de $8 \mathrm{~mm}$ de diámetro marca Kavalier cortados en tamaños de $33 \mathrm{~mm}$ de longitud. La limpieza de los tubos se realizó con hidróxido de sodio al $2 \%$ y $70{ }^{\circ} \mathrm{C}$, posteriormente con ácido nítrico al $1.5 \%$ y $60{ }^{\circ} \mathrm{C}$ durante 20 y 15 minutos respectivamente, enjuagando con agua destilada en cada ocasión. El secado de los tubos se realizó a temperatura ambiente antes de iniciar el revestimiento con el semiconductor (Rodríguez et al. 2019).

Se utilizó $\mathrm{TiO}_{2}$ en polvo en fase anatasa, marca Sigma Aldrich en dos tamaños de partícula de $25 \mathrm{y}$ $300 \mathrm{~nm}$. La suspensión de $\mathrm{TiO}_{2}$ se preparó con $2.5 \mathrm{~g}$ en $400 \mathrm{ml}$ de agua destilada y se mantuvo en agitación hasta antes de sumergir los tubos para evitar la sedimentación del catalizador (Barton et al. 2016). Para sumergir los tubos en la suspensión de catalizador, se fabricó un dispositivo de cloruro de polivinilo (PVC), con la finalidad de introducir en la suspensión 25 tubos a la vez por tres ciclos. La separación de los tubos de suspensión se llevó a cabo retirando la suspensión por sifón mediante un tubo de vidrio con válvula de control de flujo para regular la velocidad de separación. El tiempo de secado al ambiente fue de 30 min y posteriormente se llevó al horno por $1 \mathrm{~h}$ a $110^{\circ} \mathrm{C}$. Finalmente se introdujo a la mufla por $1 \mathrm{~h}$ a $400{ }^{\circ} \mathrm{C}$ para asegurar la adhesión del revestimiento de $\mathrm{TiO}_{2}$ al sustrato de vidrio. Este procedimiento se aplicó de forma separada para las partículas de $\mathrm{TiO}_{2}$ de $25 \mathrm{~nm}$ y de $300 \mathrm{~nm}$. Los tubos fueron enfriados y pesados para determinar la cantidad de fotocatalizador por milímetro cuadrado de sustrato. Para realizar el cálculo se pesaron los tubos antes y después del revestimiento con el fin de conocer la masa adherida de $\mathrm{TiO}_{2}$. El área de cada tubo se obtuvo de la geometría de un cilindro considerando la superficie exterior e interior. Se realizaron tres ciclos de revestimiento para cada tamaño de partícula y posteriormente se determinaron las masas por cada ciclo para conocer la secuencia de acumulación del fotocatalizador sobre el sustrato de borosilicato.

\section{Caracterización del revestimiento de $\mathrm{TiO}_{2}$}

La microestructura de las muestras se analizó mediante difracción de rayos $\mathrm{X}$, utilizando un difractómetro Ultima IV (Rigaku) operado a $40 \mathrm{kV}$ y $30 \mathrm{~mA}$, con un ánodo de $\mathrm{Cu}\left(\lambda_{\mathrm{Ka}}=0.154 \mathrm{~nm}\right)$. La medición se realizó en el intervalo $2 \mathrm{q}$ de 10 a $60^{\circ}$, con un tamaño de paso de $0.02^{\circ}$ y una velocidad de escaneo de $1 \%$ min. Los tamaños de partícula de los precursores se determinaron por DLS, utilizando un analizador de nanopartículas Nanoplus (Nanometrix). La morfología de la superficie y el área efectiva de contacto del revestimiento depositado se obtuvo mediante microscopia de fuerza atómica (AFM, por sus siglas en inglés) usando un equipo XE7 (Park Systems) operado en modo dinámico (tapping). E1 análisis del revestimiento depositado en el sustrato se obtuvo de los datos de las imágenes obtenidas mediante AFM (Krumdieck et al. 2017) y es un referente útil en el proceso de adsorción. Las imágenes 
se obtuvieron con el software XEI 4.3.0. Build 5. La resolución de la imagen se obtuvo a $256 \times 256$ puntos por línea sobre una superficie de $5 \times 5 \mu \mathrm{m}$.

\section{Oxidación fotocatalítica de PBC}

Los ensayos fotocatalíticos se realizaron en tubos de $10 \times 120 \mathrm{~mm}$ con tapa roscada. Dentro de cada tubo de reacción se colocaron dos piezas de borosilicato con $\mathrm{TiO}_{2}$ inmovilizado. La solución indicadora de púrpura de bromocresol ( $\mathrm{PBC}, \mathrm{C}_{21} \mathrm{H}_{16} \mathrm{Br}_{2} \mathrm{O}_{5} \mathrm{~S}$, $\mathrm{MW}=540.24 \mathrm{~g} / \mathrm{mol}$ ), se preparó en concentraciones de $5,10,20$ y $30 \mathrm{ppm}$ a un $\mathrm{pH}$ de 9.5 . Se construyó una caja de acrílico recubierto de papel de aluminio dentro de la cual se colocó la lámpara UV $\left(\lambda_{\max }=254\right.$ $\mathrm{nm}$ ) de $25 \mathrm{~W}$ (Evans), protegida por un portalámpara de borosilicato. Los tubos con $\mathrm{PBC}$ se expusieron a luz UV dentro de la caja de acrílico en dos series, la primera con partículas de $\mathrm{TiO}_{2}$ de $25 \mathrm{~nm}$ y la segunda con partículas de $300 \mathrm{~nm}$ identificadas como PBC25 y $\mathrm{PBC} 300$, respectivamente.

Las pruebas fotocatalíticas se llevaron a cabo a temperatura ambiente. Se colocaron dos testigos, el primero con solución de $\mathrm{PBC}$ de $20 \mathrm{ppm}$ y un par de tubos revestidos de $\mathrm{TiO}_{2}$ aislado de la luz, y el segundo con solución de PBC de 20 ppm sin fotocatalizador para observar efectos fotolíticos sobre la solución de PBC.

\section{Medición de la actividad fotocatalítica}

Dado que la fotocatálisis es un fenómeno de superficie, la solución de PBC se mantuvo en contacto con la capa de $\mathrm{TiO}_{2}$ por un periodo de $30 \mathrm{~min}$ para permitir el proceso de adsorción. Mediante la medición de la absorbancia a una longitud de onda de referencia $(580 \mathrm{~nm})$ antes y después del contacto, se obtuvo la cantidad adsorbida de PBC sobre el fotocatalizador (Pala et al. 2020). La actividad fotocatalítica de los tubos revestidos con $\mathrm{TiO}_{2}$ fue evaluada por la degradación de $\mathrm{PBC}$, realizando lecturas de absorbancia a $580 \mathrm{~nm}$ en periodos de 30 min durante un lapso de $3 \mathrm{~h}$. La absorbancia de la solución de $\mathrm{PBC}$ se realizó en un espectrofotómetro UV-vis DU 730 (Beckman Coulter). El porcentaje de degradación de $\mathrm{PBC}$ se utilizó como indicador para evaluar y comparar el desempeño fotocatalítico del catalizador inmovilizado en los tubos de vidrio.

El porcentaje de degradación se calculó de acuerdo con la concentración inicial $\left(C_{0}\right)$ y la concentración $C$ en el tiempo $t$ de PBC, como se indica en la ecuación 2:

Degradación $(\%)=\frac{\left(C_{0}-C_{t}\right) \times 100}{C_{0}}$
La cinética de reacción de degradación del púrpura de bromocresol se comparó con la cinética de reacción de pseudoprimer orden, la cual puede expresarse como:

$\frac{d[C]}{d t}=-K[C]$

Al integrar en los límites se obtiene:

$\ln \left(\frac{C_{0}}{C}\right)=K_{\text {app }} t$

donde $K_{\text {app }}$ es la constante de velocidad aparente del modelo de pseudoprimer orden $\left(\mathrm{min}^{-1}\right), C_{0}$ es la concentración inicial en partes por millón (ppm) de $\mathrm{PBC}$ y $C$ es la concentración de $\mathrm{PBC}$ al tiempo $t$ (min). Mientras que el porcentaje de variación de la gráfica de $\ln \left(\mathrm{C}_{0} / C\right)$ contra $t$ sea mayor a 0.95 , los datos experimentales estarán correctamente ajustados al modelo propuesto (Jiang et al. 2013, Bouarioua y Zerdaoui 2017).

\section{RESULTADOS Y DISCUSIÓN}

\section{Microestructura del $\mathrm{TiO}_{2}$ impregnado en tubos}

La cantidad de catalizador adherido a los tubos de borosilicato se cuantificó por diferencia de pesos de los tubos antes y después del revestimiento por inmersión de $\mathrm{TiO}_{2}$, el cual fue incrementándose con el número de capas como se muestra en el cuadro I. La velocidad de separación de los tubos del medio fue de $0.064 \mathrm{~mm} / \mathrm{s} \mathrm{y}$ la cantidad fijada de $\mathrm{TiO}_{2}$ para el tamaño de partícula de 25 y $300 \mathrm{~nm}$ después de tres revestimientos fue de 3.210 y $0.736 \mathrm{mg} / \mathrm{cm}^{2}$, respectivamente. Considerando la geometría de un cilindro y sus dimensiones $(3.2 \times .2$ cada tubo de u cm de largo, diámetro exterior, diámetro interior y espesor, respectivamente), se tiene que el área total de cada tubo es de $14.514 \mathrm{~cm}^{2}$.

La fase cristalina del $\mathrm{TiO}_{2}$ de 25 y $300 \mathrm{~nm}$, obtenida mediante DRX, se puede observar en la figura 1. Los picos en la difracción corresponden a la fase anatasa (tarjeta PDF No. 021-1272) para los dos tamaños de partícula. Con este resultado se confirman tanto la fase cristalina como que el tratamiento térmico en el revestimiento no promovió el cambio de ésta. También se puede observar que la cristalinidad aumenta conforme se incrementa el tamaño de partícula, lo cual se refleja en la intensidad de los picos en ángulos de difracción. Los tamaños de partícula de los precursores obtenidos por DLS fueron de $25 \mathrm{y}$ 300 nm (PBC25 y PBC300, respectivamente).

En la actividad fotocatalítica, el área efectiva de contacto del $\mathrm{TiO}_{2}$ inmovilizado con las moléculas 
CUADRO I. MASA FIJADA DE DIÓXIDO DE TITANIO POR NÚMERO DE CAPA MEDIANTE EL MÉTODO DE REVESTIMIENTO POR INMERSIÓN.

\begin{tabular}{ccccc}
\hline $\begin{array}{c}\text { Tamaño de } \\
\text { partícula } \\
(\mathrm{nm})\end{array}$ & $\begin{array}{c}\text { Número } \\
\text { de } \\
\text { capa }\end{array}$ & $\begin{array}{c}\text { Masa } \\
\text { fijada } \\
(\mathrm{mg})\end{array}$ & $\begin{array}{c}\text { Masa } \\
\text { acumulada } \\
(\mathrm{mg})\end{array}$ & $\begin{array}{c}\text { Masa acumulada/ } \\
\text { área de superficie } \\
\left(\mathrm{mg} / \mathrm{cm}^{2}\right)\end{array}$ \\
\hline 25 & 1 & 32.6 & 32.6 & 2.246 \\
25 & 2 & 11.6 & 44.2 & 3.044 \\
25 & 3 & 2.4 & 46.6 & 3.210 \\
300 & 1 & 5.7 & 5.7 & 0.392 \\
300 & 2 & 1.2 & 6.9 & 0.474 \\
300 & 3 & 3.8 & 10.7 & 0.736 \\
\hline
\end{tabular}

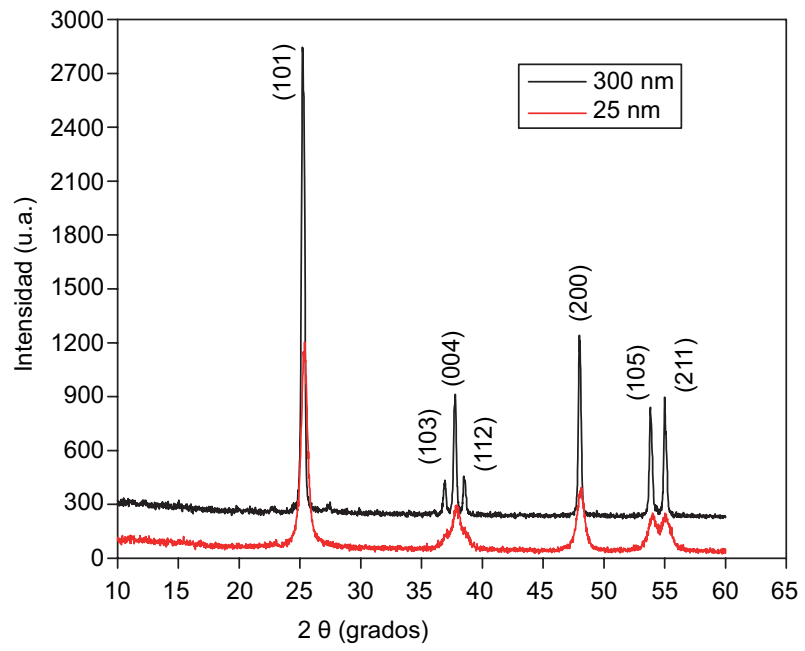

Fig. 1. Patrón de difracción de rayos x (DRX) de $\mathrm{TiO}_{2}$ en fase anatasa en partículas de 25 y $200 \mathrm{~nm}$. del PBC es el parámetro importante a seguir, ya que puede orientar sobre los resultados de degradación esperados. Mediante AFM se estimaron áreas totales efectivas de 5.171 y $3.256 \mu \mathrm{m}^{2}$ para partículas de 25 $\mathrm{nm}$ y $300 \mathrm{~nm}$, respectivamente, en un área total de $25 \mu \mathrm{m}^{2}$ de barrido en el sustrato. De esta forma se estableció que hay mayor fotoactividad en PBC25 que en PBC300. El área efectiva de contacto del fotocatalizador inmovilizado puede conducir a un mayor número de grupos OH- adsorbidos para la formación de radicales, así como de la adsorción de moléculas de PBC para el inicio de la reacción, como se establece en la primera etapa del modelo de isotermas de Langmuir.

En la figura 2, se puede observar la morfología de la superficie del $\mathrm{TiO}_{2}$ inmovilizado en borosilicato por el método de revestimiento por inmersión, correspondiente a tamaños de partícula de 25 y 300
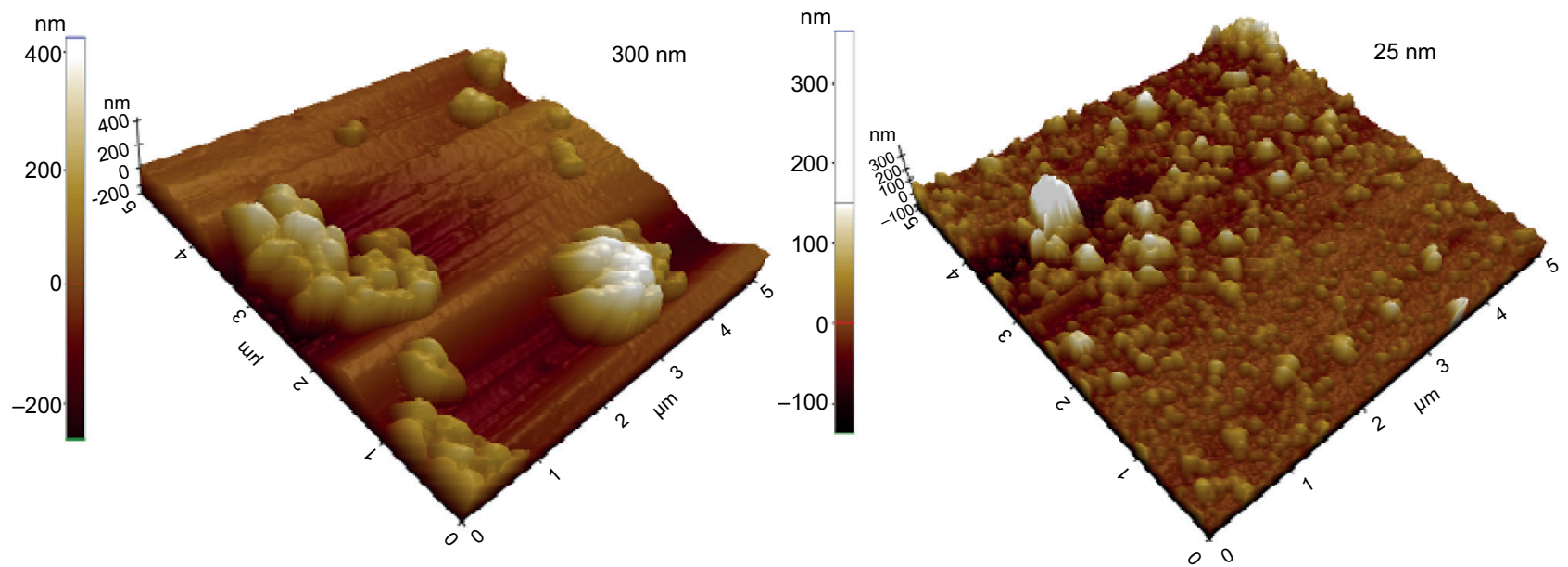

Fig. 2. AFM de la morfología de la superficie de $\mathrm{TiO}_{2}$ fijado sobre borosilicato en tamaños de 300 y $25 \mathrm{~nm}$. 
$\mathrm{nm}$, respectivamente, obtenidas mediante AFM en un área de $5 \times 5 \mu \mathrm{m}$.

\section{Efecto de la concentración inicial de PBC}

En la figura 3 se puede observar el efecto de la concentración inicial de $\mathrm{PBC}$ sobre el porcentaje de degradación en un periodo de $3 \mathrm{~h}$ de irradiación en la unidad experimental. Para un mismo tamaño de partícula, la tendencia de la degradación es decreciente conforme se incrementa la concentración de PBC. Este fenómeno se debe a la disminución de la cantidad de luz que incide sobre la superficie inmovilizada de $\mathrm{TiO}_{2}$, lo que reduce a su vez la formación de pares iniciadores del proceso de oxidación, como se destaca en la concentración de $30 \mathrm{ppm}$ de PBC. Esta disminución resulta de la absorción de fotones por la solución de PBC. Entre más concentrada sea ésta, menor será la cantidad de luz transmitida que podrá incidir en la superficie de $\mathrm{TiO}_{2}$, de acuerdo con lo establecido en la ley de Lambert-Beer. Por el contrario, cuando la concentración es baja (de 5 o 10 ppm de PBC) la alta incidencia de luz UV sobre la superficie del $\mathrm{TiO}_{2}$ inmovilizado es consecuencia de la baja absorbancia de la solución, permitiendo un incremento en la formación de pares respecto a concentraciones elevadas.

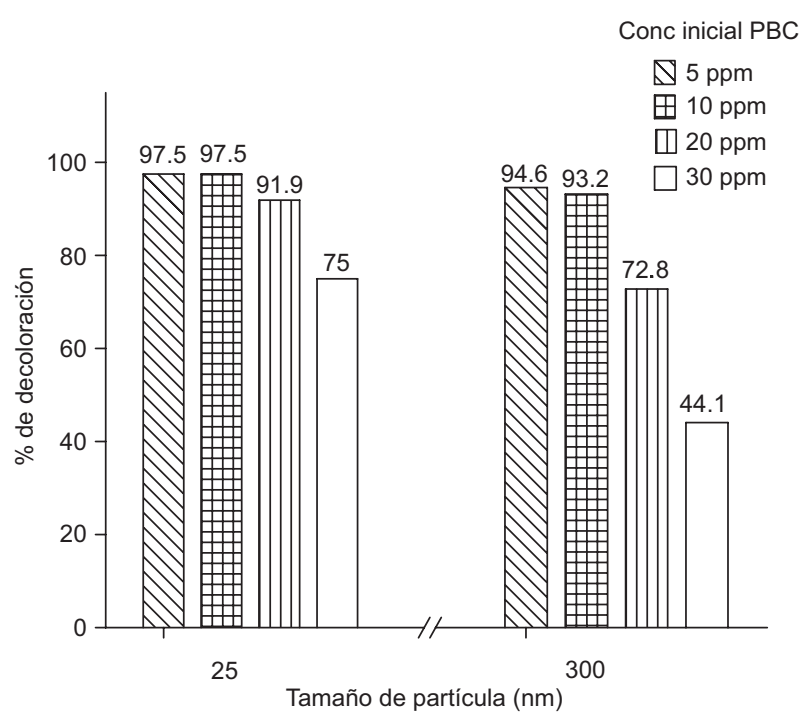

Fig. 3. Decoloración de $\mathrm{PBC}$ en función del tamaño de partícula de $\mathrm{TiO}_{2}$.

\section{Efecto del tamaño de partícula de $\mathrm{TiO}_{2}$}

La actividad fotocatalítica de PBC se estudió en dos tamaños de partícula de $\mathrm{TiO}_{2}$ en su fase anatasa, inmovilizadas en borosilicato por el método de revestimiento por inmersión. Como se observa en la figura 4, los porcentajes de degradación de $\mathrm{PBC}$ respecto al tamaño de partícula del fotocatalizador y la correspondiente concentración de PBC obtenidos fueron los siguientes: 97.5 y $94.6 \%$ a 5 ppm, 97.5 y $93.2 \%$ a $10 \mathrm{ppm}, 91.9$ y $72.8 \%$ a $20 \mathrm{ppm}$ y 75 , y $44.1 \%$ a 30 ppm en PBC25 y PBC300, respectivamente. La exposición a luz ultravioleta se realizó en un lapso de $3 \mathrm{~h}$, observándose en los resultados que la tasa de degradación en PBC25 es más elevada que en $\mathrm{PBC} 300$. A concentraciones de 5 y $10 \mathrm{ppm}$, la diferencia de degradación observada entre los dos tamaños no es significativa, aunque puede apreciarse que para el caso de 5 ppm se alcanza el equilibrio en el lapso de $3 \mathrm{~h}$ en $\mathrm{PBC} 25$. Por otro lado, a concentraciones de 20 y $30 \mathrm{ppm}$ de $\mathrm{PBC}$ se obtuvo una diferencia de porcentaje de 19.1 y $30.9 \%$ de degradación mayor en PBC25. En la transformación del 2-clorofenol, se demostró que la velocidad de degradación fotocatalítica es más rápida cuando se reduce el tamaño de partícula (Lin et al. 2006), tal como ocurrió en el proceso de degradación de $\mathrm{PBC}$ en los tamaños de 25 y $300 \mathrm{~nm}$.

En la dinámica del proceso de recombinación electrón-hueco, el tamaño de partícula es un factor crucial. Al reducir el tamaño de partícula también se incrementa el área de superficie, con lo cual aumentan los sitios activos disponibles de la superficie. Una disminución en el tamaño de partícula debería permitir una elevada eficiencia fotónica con una tasa de transferencia de cargas alta; por lo tanto, en partículas nanocristalinas, cuando el tamaño es extremadamente pequeño, el proceso de recombinación llega a ser importante. En el régimen ultrafino, la mayoría de los pares e-h generados están cerca de la superficie, por lo que se someten a una rápida recombinación, principalmente debido a la abundancia de sitios atrapadores y a la carencia de fuerzas de control para la separación de pares. De lo anterior se deduce que la recombinación de cargas no fue un factor que afectara al fotocatalizador inmovilizado, dado que las partículas de $25 \mathrm{~nm}$ no son suficientemente pequeñas para afectar los sitios activos disponibles.

\section{Modelo cinético de degradación}

El modelo cinético de degradación de PBC obtenido fue de pseudoprimer orden para $\mathrm{PBC} 25$ y PBC300, y puede describirse con el modelo de Langmuir para concentraciones bajas del indicador:

$r=\frac{-d C}{d T}=\frac{K k_{r} C}{1+K C}$ 


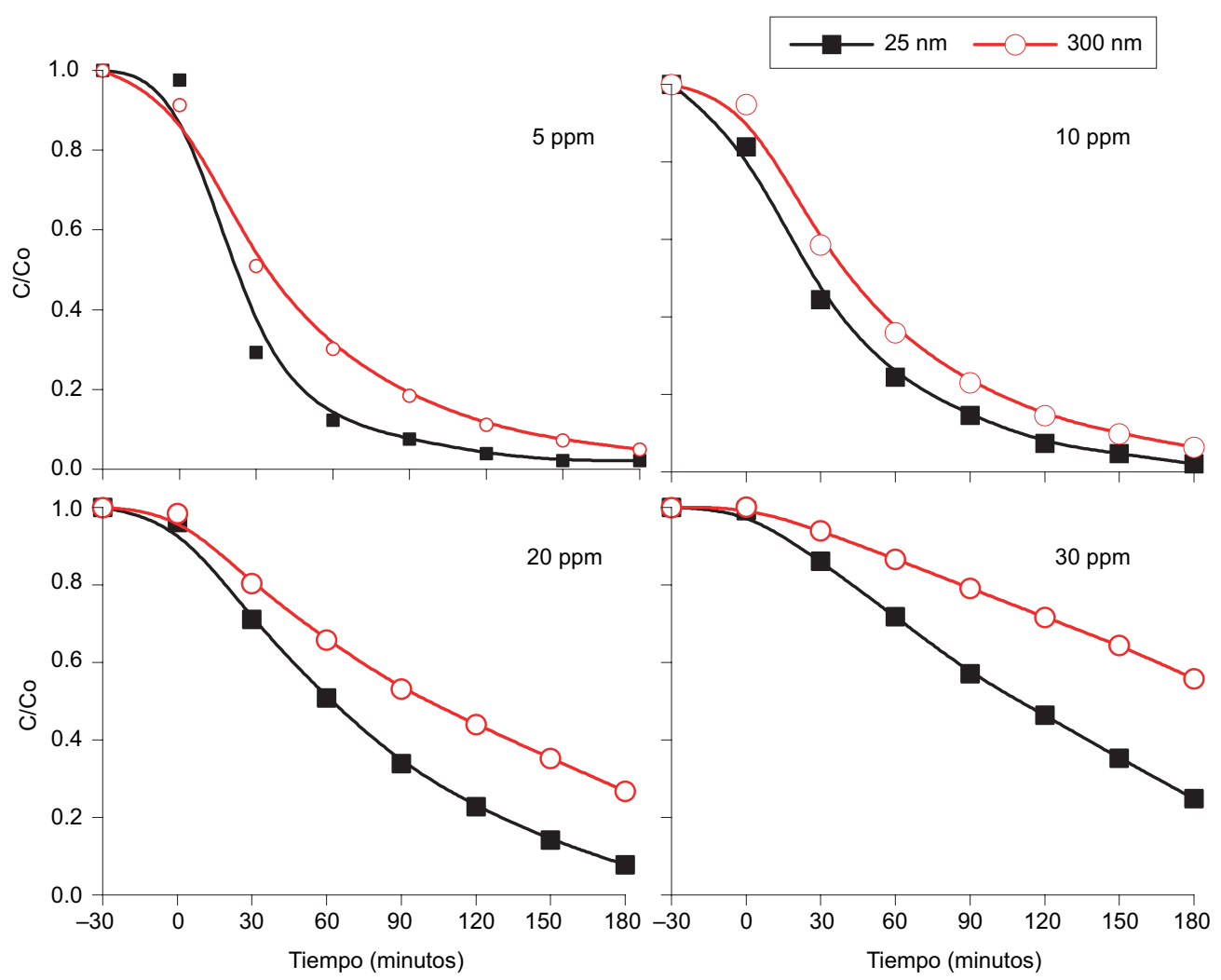

Fig. 4. Efecto del tamaño de partícula del fotocatalizador a diferente concentración inicial de PBC.

donde $r$ representa la tasa de degradación, $K$ es la constante de equilibrio de adsorción de $\mathrm{PBC}$ sobre la superficie del catalizador y $k_{\mathrm{r}}$ la constante cinética de la reacción de degradación. Integrando la ecuación 5 para concentraciones bajas de $\mathrm{PBC}$, el término es muy pequeño, por lo que el denominador tiende a la unidad y se obtiene la ecuación 4 . Al graficar esta última se obtiene la linealidad de $\ln \left(C_{0} / C\right)$ contra $t$, como se observa en la figura 5. Los valores de la constante de velocidad aparente en PBC25 fueron de 0.7132, $0.5961,0.4124$ y $0.2277 \mathrm{~min}^{-1}$, y para PBC300 de $0.0162,0.0149,0.0071$ y 0.0032 en las concentraciones de 5, 10, 20 y 30 ppm de PBC, respectivamente. La diferencia entre las magnitudes de las pendientes de PBC25 y PBC300 se debe básicamente a dos factores: 1) la mayor área de superficie de $\mathrm{PBC} 25$ como consecuencia del tamaño de partícula, lo que conduce a un mayor número de sitios activos sobre la superficie disponible para la adsorción de PBC, y 2) se considera que la cantidad de luz UV que llega a la superficie de $\mathrm{TiO}_{2}$ es una función de la concentración del indicador, por lo que a menor concentración de $\mathrm{PBC}$ es mayor la cantidad de luz que interacciona con el fotocatalizador, lo que maximiza la formación de pares en 5 y 10 ppm de PBC. Por el contrario, a mayor concentración de $\mathrm{PBC}$, la cantidad de luz que llega al fotocatalizador desciende al ser absorbida por el indicador, disminuyendo en consecuencia la formación de especies reactivas en 20 y 30 ppm. En el análisis de la degradación fotocatalítica del anaranjado de metilo se reportó que la disminución de degradación de la molécula modelo a altas concentraciones se debe probablemente al efecto de absorción de la luz UV por dicha molécula (Niu y Hao 2013); éste disminuye la transmitancia de la luz por la solución coloreada, aminorando los fotones que pueden alcanzar la superficie del catalizador. Otra presunta razón es que el número de oxidantes generados en solución es fijo, lo que resulta en un bajo porcentaje de degradación del indicador a alta concentración de PBC. Se cree que la degradación fotocatalítica del indicador se facilita mediante la adición de aceptores de electrones como el peróxido de hidrógeno $\left(\mathrm{H}_{2} \mathrm{O}_{2}\right)$.

En la degradación fotocatalítica a diferentes concentraciones iniciales de verde de metilo (Hadjltaief et al. 2016) sobre catalizadores $\mathrm{ZnO} / \mathrm{TiO}_{2}$ soportados, la eficiencia de degradación se redujo conforme la concentración inicial de verde de metilo 


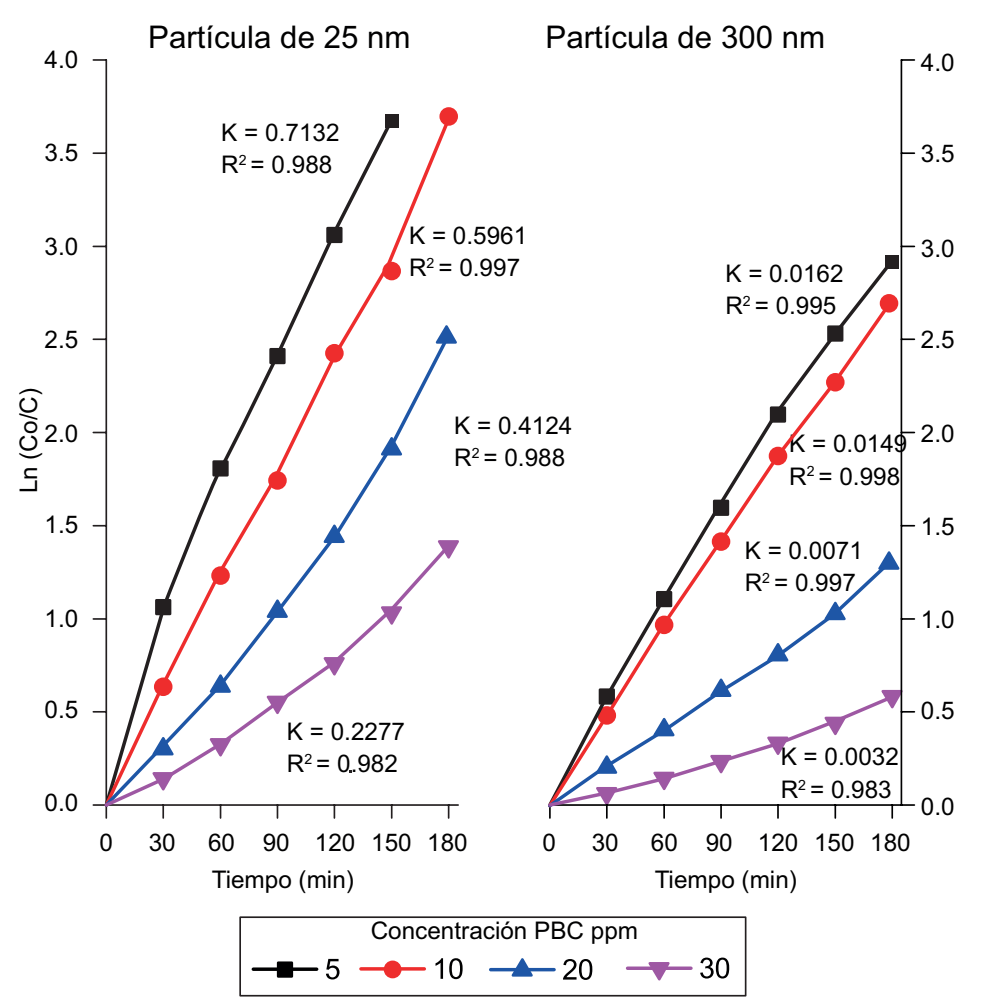

Fig. 5. Constantes de velocidad de decoloración en diferente concentración inicial de PBC y 2 tamaños de partícula.

se incrementó. Los autores indicaron que una elevada concentración del color produce una gran cantidad de moléculas de color adsorbidas sobre la superficie del catalizador, bloqueando los sitios activos del mismo.

\section{Adsorción y fotólisis de púrpura de bromocresol}

Se evaluó la adsorción del indicador PBC sobre el revestimiento de $\mathrm{TiO}_{2}$ en un periodo de $30 \mathrm{~min}$ manteniendo sin encender la lámpara de luz UV. En la figura 6 se observa la cantidad adsorbida de PBC sobre $\mathrm{TiO}_{2}$ como una función de la concentración inicial y del tamaño de partícula. En la misma figura se observa la muestra testigo 1, que fue aislada de la luz y no experimentó degradación alguna (excepto la del proceso de adsorción inicial), en tanto que en la muestra testigo 2, la cual se expuso a la luz y 20 ppm de PBC sin catalizador, se observa el fenómeno de fotólisis. Sin embargo, como se observa en la gráfica, el porcentaje del proceso de degradación es mayor cuando el experimento se lleva a cabo con el fotocatalizador y luz UV.

Las muestras testigo alcanzaron 2.2 y $31.4 \%$ de degradación para los testigos 1 y 2 , respectivamente. Para el anaranjado de metilo se han obtenido

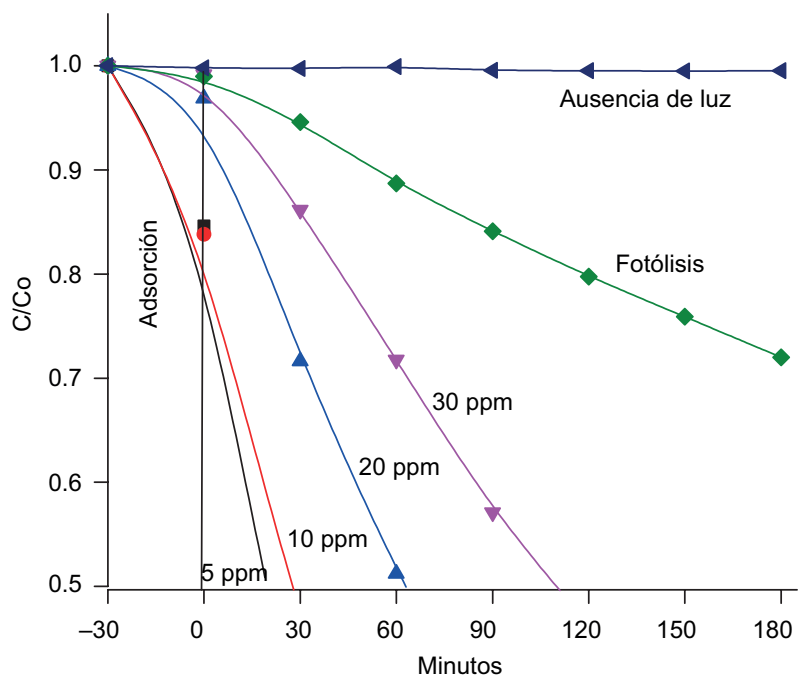

Fig. 6. Fotólisis y ausencia de luz en 20 ppm de PBC. Adsorción en superficie de $\mathrm{TiO}_{2}$ en concentraciones de 5, 10, 20 y 30 ppm de PBC.

resultados que van de 2.6 a $5.3 \%$ de degradación, dependiendo de la intensidad de la radiación UV a que se somete el proceso (Haji et al. 2011). 
Sobre el estudio de fotólisis al PBC y verde de metilo de Bousnoubra et al. (2016) con concentración de $25 \mathrm{ppm}$ mediante un reactor tubular aireado e irradiación UV de $254 \mathrm{~nm}$ en un lapso de $240 \mathrm{~min}$, estos autores obtuvieron 25 y $66 \%$ de degradación para $\mathrm{PBC}$ y verde de metilo, respectivamente, y concluyeron que el verde de metilo absorbe mejor la luz que el PBC. En condiciones oscuras, sus resultados experimentales preliminares no demostraron degradación de ambos indicadores.

\section{CONCLUSIONES}

Mediante DRX se confirmó que la estructura cristalina de $\mathrm{TiO}_{2}$ para $\mathrm{PBC} 25$ y $\mathrm{PBC} 300$ corresponde a la fase anatasa pura, destacando la cristalinidad por la intensidad de los picos en la partícula de mayor tamaño. La morfología de la superficie obtenida por AFM en PBC25 confirmó un mayor número de granos que en $\mathrm{PBC} 300$, lo cual promueve un mayor número de moléculas adsorbidas en la superficie PBC25. A su vez, el área total de contacto de moléculas de PBC con el fotocatalizador fue mayor en $58.8 \%$ en PBC25 que en $\mathrm{PBC} 300$, lo que a su vez origina una mayor degradación en las partículas de $25 \mathrm{~nm}$.

La cinética de degradación fotocatalítica de PBC en $\mathrm{TiO}_{2}$ inmovilizado es de primer orden para las concentraciones analizadas de PBC. Los modelos del proceso de adsorción son congruentes con el modelo de Langmuir a concentraciones bajas para 5 y 10 ppm y a concentraciones altas para 20 y $30 \mathrm{ppm}$. El incremento de la concentración inicial a $30 \mathrm{ppm}$ de PBC redujo la tasa de reacción de fotodegradación a causa de la absorción de luz en la solución, provocando el descenso de formación de pares requeridos para iniciar la oxidación fotocatalítica. Las partículas de $25 \mathrm{~nm}$ fijadas son de mayor eficiencia en la fotodegradación que las de $300 \mathrm{~nm}$. El tamaño óptimo de partícula es el que se obtiene de la competencia de la eficiencia de absorción y dispersión de luz, dinámica de cargas y área de superficie. La oxidación fotocatalítica de materia orgánica en superficies inmovilizadas resulta de gran interés como opción para el tratamiento de agua residual, sobre todo porque no se requiere un tratamiento adicional de recuperación del fotocatalizador.

\section{AGRADECIMIENTOS}

El primer autor agradece el apoyo del Consejo Nacional de Ciencia y Tecnología (CONACYT) por la beca otorgada para cursar el doctorado en alimentos y biotecnología dentro del programa de doctorado del Instituto Tecnológico de Tuxtla Gutiérrez.

\section{REFERENCIAS}

Ali I., Peng C., Naz I., Lin D., Saroj D.P. y Ali M. (2019). Development and application of novel bio-magnetic membrane capsules for the removal of the cationic dye malachite green in wastewater treatment. RSC Adv. 9 (7), 3625-3646. https://doi.org/10.1039/c8ra09275c

Bagheri S., TermehYousefi A. y Do T. (2017). Photocatalytic pathway toward degradation of environmental pharmaceutical pollutants: Structure, kinetics and mechanism approach. Catal. Sci. Technol. 7 (20), 45484569. https://doi.org/10.1039/C7CY00468K

Barton I., Matejec V. y Matousek J. (2016). Photocatalytic activity of nanostructured $\mathrm{TiO}_{2}$ coating on glass slides and optical fibers for methylene blue or methyl orange decomposition under different light excitation. J. Photoch. Photobio. A 317, 72-80. https://doi.org/10.1016/j. jphotochem.2015.11.009

Binaeian E., Seghatoleslami N., Chaichi M.J. y Tayebi H.-A. (2016). Preparation of titanium dioxide nanoparticles supported on hexagonal mesoporous silicate (HMS) modified by oak gall tannin and its photocatalytic performance in degradation of azo dye. Adv. Powder Technol. 27 (4), 1047-1045. https://doi. org/10.1016/j.apt.2016.03.012

Bouarioua A. y Zerdaoui M. (2017). Photocatalytic activities of $\mathrm{TiO}_{2}$ layers immobilized on glass substrates by dip-coating technique toward the decolorization of methyl orange as a model organic pollutant. Journal of Environmental Chemical Engineering 5 (2), 15651574. https://doi.org/10.1016/j.jece.2017.02.025

Bousnoubra I., Djebbar K., Abdessemed A. y Sehili T. (2016). Decolorization of methyl green and bromocresol purple in mono and binary systems by photochemical processes: Direct UV photolysis, Acetone/UV and $\mathrm{H}_{2} \mathrm{O}_{2} / \mathrm{UV}$. A comparative study. Desalin. Water Treat. 57 (57), 27710-27725. https://doi.org/10.1080/19443 994.2016.1174741

Bousnoubra I., Sehili T. y Djebbar K. (2017). Treatment of bromocresol purple dye by several photochemical processes in aqueous medium: A comparative study. Der Pharma Chemica 9 (5), 13-17.

Carneiro J.T., Savenije T.J., Moulijn J.A. y Mul G. (2010). Toward a physically sound structure-activity relationship of $\mathrm{TiO}_{2}$-based photocatalysts. J. Phys. Chem. C 114 (1), 327-332. https://doi.org/10.1021/jp906395w

Chen W.-F., Koshy P. y Sorrell C.C. (2016). Effects of film topology and contamination as a function of thickness 
on the photo-induced hydrophilicity of transparent $\mathrm{TiO}_{2}$ thin films deposited on glass substrates by spin coating. J. Mater Sci. 51 (5), 2465-2480. https://doi. org/10.1007/s10853-015-9559-y

Das R., Vecitis C.D., Schulze A., Cao B., Ismail A.F., Lu X., Chen J. y Ramakrishna S. (2017). Recent advances in nanomaterials for water protection and monitoring. Chem. Soc. Rev. 46 (22), 6946-7020. https://doi. org/10.1039/c6cs00921b

Deng W., Pan F., Batchelor B., Jung B., Zhang P., AbdelWahab A., Zhou H. y Li Y. (2019). Mesoporous $\mathrm{TiO}_{2}-\mathrm{BiOBr}$ microspheres with tailorable adsorption capacities for photodegradation of organic water pollutants: probing adsorption-photocatalysis synergy by combining experiments and kinetics modeling. Environ. Sci.-Wat. Res. 5 (4), 769-781. https://doi. org/10.1039/C8EW00922H

Du L., Coyle T.W., Chien K., Pershin L., Li T. y Golozar M. (2015). Titanium dioxide coating prepared by use of a suspension-solution plasma-spray process. J. Therm. Spray Techn. 24 (6), 915-924. https://doi.org/10.1007/ s11666-015-0251-1

González-Casamachín D.A., Rivera de la Rosa J., Lucio Ortiz C.J., De Haro De Rio D.A., Martínez-Vargas D.X., Flores-Escamilla G.A., Dávila-Guzmán N.E., OvandoMedina V.M. y Moctezuma-Velázquez E. (2019). Visible-light photocatalytic degradation of acid violet 7 dye in a continuous annular reactor using $\mathrm{ZnO} / \mathrm{PPy}$ photocatalyst: Synthesis, characterization, mass transfer effect evaluation and kinetic analysis. Chem. Eng. J. 373, 325-337. https://doi.org/10.1016/j.cej.2019.05.032

Gora S., Sokolowski A., Hatat-Fraile M., Liang R., Zhou Y.N. y Andrews S. (2018). Solar photocatalysis with modified $\mathrm{TiO}_{2}$ photocatalysts: Effects on NOM and disinfection byproduct formation potential. Environ. Sci.Wat. Res. 4 (9), 1361-1376, https://doi.org/10.1039/ c8ew00161h.

Gupta V. K., Jain R., Mittal A., Saleh T.A., Nayak A., Agarwal S. y Sikarwar S. (2012). Photo-catalytic degradation of toxic dye amaranth on $\mathrm{TiO}_{2} / \mathrm{UV}$ in aqueous suspensions. Mater. Sci. Eng. C-Mater. 32 (1), 12-17. https://doi.org/10.1016/j.msec.2011.08.018

Habibi M.H. y Mosavi V. (2017). Photo-catalytic activity of highly efficient binary $\mathrm{Mn}-\mathrm{Fe}$ nano composite oxides for degradation of cresol fast violet: phase formation and band gap study. J. Mater. Sci-Mater. El. 28 (18), 13643-13648. https://doi.org/10.1007/ s10854-017-7206-x

Hadjltaief H.B., Zina M.B., Gálvez M.E. y da Costa P. (2016). Photocatalytic degradation of methyl green dye in aqueous solution over natural clay-supported ZnO-TiO 2 . J. Photoch. Photobio. A 315, 25-33. https:// doi.org/10.1016/j.jphotochem.2015.09.008
Haji S., Benstaali B. y al-Bastaki N. (2011). Degradation of methyl orange by $\mathrm{UV} / \mathrm{H}_{2} \mathrm{O}_{2}$ advanced oxidation process. Chem. Eng. J. 168 (1), 134-139. https://doi. org/10.1016/j.cej.2010.12.050

Henderson M.A. (2011). A surface science perspective on $\mathrm{TiO}_{2}$ photocatalysis. Surf. Sci. Rep. 66 (6), 185-297. https://doi.org/10.1016/j.surfrep.2011.01.001

Jiang W., Joens J.A., Dionysiou D.D. y O'Shea K.E. (2013). Optimization of photocatalytic performance of $\mathrm{TiO}_{2}$ coated glass microspheres using response surface methodology and the application for degradation of dimethyl phthalate. J. Photoch. Photobio. A 262, 7-13. https://doi.org/10.1016/j.jphotochem.2013.04.008

Khatibnezhad H. y Faghihi-Sani M. A. (2015). Preparation and characterization of nanostructured $\mathrm{TiO}_{2}$ thin film codoped with nitrogen and vanadium on glass surface by sol-gel dip-coating method. Res. Chem. Intermediat. 41 (10), 7349-7361. https://doi.org/10.1007/s11164014-1816-1

Kment S., Kmentova H., Hubicka Z., Olejnicek J., Cada M. y Krysa J. (2015). Enhanced photocatalytic activity of silver-doped nanoparticulate $\mathrm{TiO}_{2}$ thin films with respect to the method of doping. Res. Chem. Intermediat. 41 (12), 9343-9355. http://doi.org/10.1007/ s11164-015-2022-5

Koci K., Obalová L., Matejová L., Plachá D., Lacný Z., Jirkovský J., y Solcová O. (2009). Effect of $\mathrm{TiO}_{2}$ particle size on the photocatalytic reduction of $\mathrm{CO}_{2}$. Appl. Catal. B-Environ. 89 (3), 494-502. https://doi. org/10.1016/j.apcatb.2009.01.010

Krumdieck S., Gorthy R., Gardecka A.J., Lee D., Miya S.S., Talwar S.D., Polson M.I.J. y Bishop C. (2017). Characterization of photocatalytic, wetting and optical properties of $\mathrm{TiO}_{2}$ thin films and demonstration of uniform coating on a 3-D surface in the mass transport controlled regime. Surf. Coat. Tech. 326, 402-410. https://doi.org/10.1016/j.surfcoat.2016.11.064

Li S., Huang J., Mao J., Zhang L., He C., Chen G., Parkin I.P. y Lai Y. (2019). In vivo and in vitro efficient textile wastewater remediation by Aspergillus niger biosorbent. Nanoscale Adv. 1 (1), 168-176. https://doi. org/10.1039/c8na00132d

Lin H., Huang C.P., Li W., Ni C., Shah S.I. y Tseng Y.H. (2006). Size dependency of nanocrystalline $\mathrm{TiO}_{2}$ on its optical property and photocatalytic reactivity exemplified by 2-chlorophenol. Appl. Catal. B-Environ. 68 (1), 1-11. https://doi.org/10.1016/j.apcatb.2006.07.018

Lu D., Yang M., Kumar K.K., Wu P. y Peng K. (2018). Facile synthesis of $\mathrm{TiO}_{2}$-based nanosheets by the regulation of cationic surfactants for effective adsorptionphotocatalytic removal of high-chroma crystal violet. Adv. Powder Technol. 29 (3), 756-764. https://doi. org/10.1016/j.apt.2017.12.021 
Maghsoodi M., Jacquin C., Teychené B., Heran M., Tarabara V.V., Lesage G. y Snow S.D. (2019). Emerging investigator series: Photocatalysis for MBR effluent post-treatment: assessing the effects of effluent organic matter characteristics. Environ. Sci.-Wat. Res. 5 (3), 482-494. https://doi.org/10.1039/c8ew00734a

Malnieks K., Mezinskis G. y Pavlovska I. (2016). Effect of different dip-coating techniques on $\mathrm{TiO}_{2}$ thin film properties. Key Eng. Mater. 721, 128-132. https://doi. org/10.4028/www.scientific.net/KEM.721.128

Momina, Shahadat M. e Isamil S. (2018). Regeneration performance of clay-based adsorbents for the removal of industrial dyes: A review. RSC Adv. 8 (43), 2457124587. https://doi.org/10.1039/c8ra04290j

Monga D. y Basu S. (2019). Enhanced photocatalytic degradation of industrial dye by $\mathrm{g}-\mathrm{C}_{3} \mathrm{~N}_{4} / \mathrm{TiO}_{2}$ nanocomposite: Role of shape of $\mathrm{TiO}_{2}$. Adv. Powder Technol. 30 (5), 1089-1098. https://doi.org/10.1016/j. apt.2019.03.004.

Nam Y., Li L., Lee J.Y. y Prezhdo O.V. (2018). Size and shape effects on charge recombination dynamics of $\mathrm{TiO}_{2}$ nanoclusters. J. Phys. Chem. C 122 (9), 52015208. https://doi.org/10.1021/acs.jpcc.8b00691

Niu P. y Hao J. (2013). Photocatalytic degradation of methyl orange by titanium dioxide-decatungstate nanocomposite films supported on glass slides. Colloid. Surface A 431, 127-132. https://doi.org/10.1016/j. colsurfa.2013.04.033

Odling G., Pong Z.Y., Gilfillan G., Pulham C.R. y Robertson N. (2018). Bismuth titanate modified and immobilized $\mathrm{TiO}_{2}$ photocatalysts for water purification: Broad pollutant scope, ease of re-use and mechanistic studies. Environ. Sci.-Wat. Res. 4 (12), 2170-2178. https://doi.org/10.1039/c8ew00568k

Pala L.P.R., Uday V., Gogoi D. y Peela N.R. (2020). Surface and photocatalytic properties of $\mathrm{TiO}_{2}$ thin films prepared by non-aqueous surfactant assisted sol-gel method. J. Environ. Chem. Eng. 8 (5), 104267. https:// doi.org/10.1016/j.jece.2020.104267

Peleyeju M.G. y Arotiba O.A. (2018). Recent trend in visible-light photoelectrocatalytic system for degradation of organic contaminants in water/wastewater. Environ. Sci.-Wat. Res. 4 (10), 1389-1411. https://doi. org/10.1039/C8EW00276B

Pellegrino F., Pellutiè L., Sordello F., Minero C., Ortel E., Hodoroaba V.-D. y Maurino V. (2017). Influence of agglomeration and aggregation on the photocatalytic activity of $\mathrm{TiO}_{2}$ nanoparticles. Appl. Catal. B-Environ. 216, 80-87. https://doi.org/10.1016/j. apcatb.2017.05.046

Rizzi V., D’Agostino F., Fini P., Semeraro P. y Cosma P. (2017). An interesting environmental friendly cleanup:
The excellent potential of olive pomace for disperse blue adsorption/desorption from wastewater. Dyes Pigments 140, 480-490, https://doi.org/10.1016/j. dyepig.2017.01.069

Rodríguez E.M., Rey A., Mena E. y Beltrán F.J. (2019). Application of solar photocatalytic ozonation in water treatment using supported $\mathrm{TiO}_{2}$. Appl. Catal. B-Environ. 254, 237-245, https://doi.org/10.1016/j. apcatb.2019.04.095

Shinde D.R., Tambade P.S, Chaskar M.G., y Gadave K.M. (2017). Photocatalytic degradation of dyes in water by analytical reagent grades $\mathrm{ZnO}, \mathrm{TiO}_{2}$ and $\mathrm{SnO}_{2}$ : A comparative study. Drink. Water Eng. Sci. 10 (2), 109-117. https://doi.org/10.5194/dwes-10-109-2017

Srikanth B., Goutham R., Narayan R.B., Ramprasath A., Gopinath K.P. y Sankaranarayanan A.R. (2017). Recent advancements in supporting materials for immobilised photocatalytic applications in wastewater treatment. J. Environ. Manage. 200, 60-78. https://doi. org/10.1016/j.jenvman.2017.05.063

Talebi S., Chaibakhsh N. y Shoeili Z. (2019). Application of nanoscale $\mathrm{ZnS} / \mathrm{TiO}_{2}$ composite for optimized photocatalytic decolorization of a textile dye. J. Appl. Res. Technol. 15 (4), 378-385. https://doi.org/10.1016/j. jart.2017.03.007

Vaez M., Moghaddam A.Z., Mahmoodi N.M. y Alijani S. (2012). Decolorization and degradation of acid dye with immobilized titania nanoparticles. Process Saf. Environ. 90 (1), 56-64. https://doi.org/10.1016/j. psep.2011.07.005

Wang Q., Gao Q., al-Enizi A.M., Nafady A. y Ma S. (2020). Recent advances in MOF-based photocatalysis: environmental remediation under visible light. Inorg. Chem. Front. 7 (2), 300-339. https://doi.org/10.1039/ c9qi01120j

Yang Z., Zhang C., Zeng G., Tan X., Wang H., Huang D., Yang K., Wei J., Ma C. y Nie K. (2020). Design and engineering of layered double hydroxide-based catalysts for water depollution by advanced oxidation processes: a review. J. Mater. Chem. A 8 (8), 41414173. https://doi.org/10.1039/C9TA13522G

Yao S., Wang J., Zhou X., Zhou S., Pu X. y Li W. (2020). One-pot low temperature synthesis of $\mathrm{BiOX} / \mathrm{TiO}_{2}$ hierarchical composites of adsorption coupled with photocatalysis for quick degradation of colored and colorless organic pollutants. Adv. Powder Technol. 31 (5), 1924-1932. https://doi.org/10.1016/j.apt.2020.02.023

Zhang W., Lan Y., Ma M., Chai S., Zuo Q., Kim K.-H. y Gao Y. (2020). A novel chitosan-vanadium-titaniummagnetite composite as a superior adsorbent for organic dyes in wastewater. Environ. Int. 142, 105798. 1-12. https://doi.org/10.1016/j.envint.2020.105798 